\title{
Images of the month 1: Trident sign and neurosarcoidosis
}

\author{
Authors: Emily Gibbons, ${ }^{A}$ Daniel Whittam, ${ }^{B}$ Anu Jacob ${ }^{C}$ and Saif Huda
}

\begin{abstract}
A 36-year-old woman presented with a subacute, relapsing myelitis, manifesting as bilateral ascending lower limb paraesthesia, partially responsive to steroids. Imaging demonstrated a longitudinal spinal cord lesion, with a unique and characteristic sign (the 'trident sign') on axial views, which is specific to a diagnosis of neurosarcoidosis. This case highlights the importance of using this feature to distinguish a longitudinal cord lesion of sarcoidosis from other differentials.
\end{abstract}

KEYWORDS: neurosarcoidosis, sarcoidosis, trident sign, myelitis

DOI: 10.7861/clinmed.2021-0596

\section{Case presentation}

A 36 -year-old woman presented with a subacute, relapsing myelitis, manifesting as bilateral ascending lower limb paraesthesia. Symptom onset was preceded by a viral illness. There was no personal or family history of neurological/ autoimmune disease. Symptom nadir was 11 days from onset and partially responsive to steroids. Magnetic resonance imaging (MRI) of the spine demonstrated a long, enhancing abnormality from $\mathrm{C} 1-\mathrm{T} 3$, involving the dorsal aspect of the spinal cord and posterolateral white matter tracts (Fig 1a). A characteristic 'trident sign' was noted on axial views (Fig 1b). ${ }^{1}$ Cerebrospinal fluid (CSF) demonstrated a pleocytosis, normal glucose and protein, and negative oligoclonal bands. Sensory-motor relapse occurred 5 weeks after steroid therapy and plasma exchange was required. Imaging demonstrated extension of the initial cord lesion. Mediastinal lymph node biopsy following positron emission tomography confirmed sarcoidosis. Respiratory and rheumatological relapses occurred while on combination immunosuppressive therapy (mycophenolate mofetil and prednisolone) leading to the addition of methotrexate.

Authors: ${ }^{\text {A }}$ clinical fellow in neuroimmunology, The Walton Centre NHS Foundation Trust, Liverpool, UK; ${ }^{B}$ neurology specialist registar, Salford Royal NHS Foundation Trust, Salford, UK; ' ${ }^{C}$ consultant neurologist, The Walton Centre NHS Foundation Trust, Liverpool, UK and Cleveland Clinic, Abu Dhabi, United Arab Emirates;

${ }^{D}$ consultant neurologist, The Walton Centre NHS Foundation Trust, Liverpool, UK
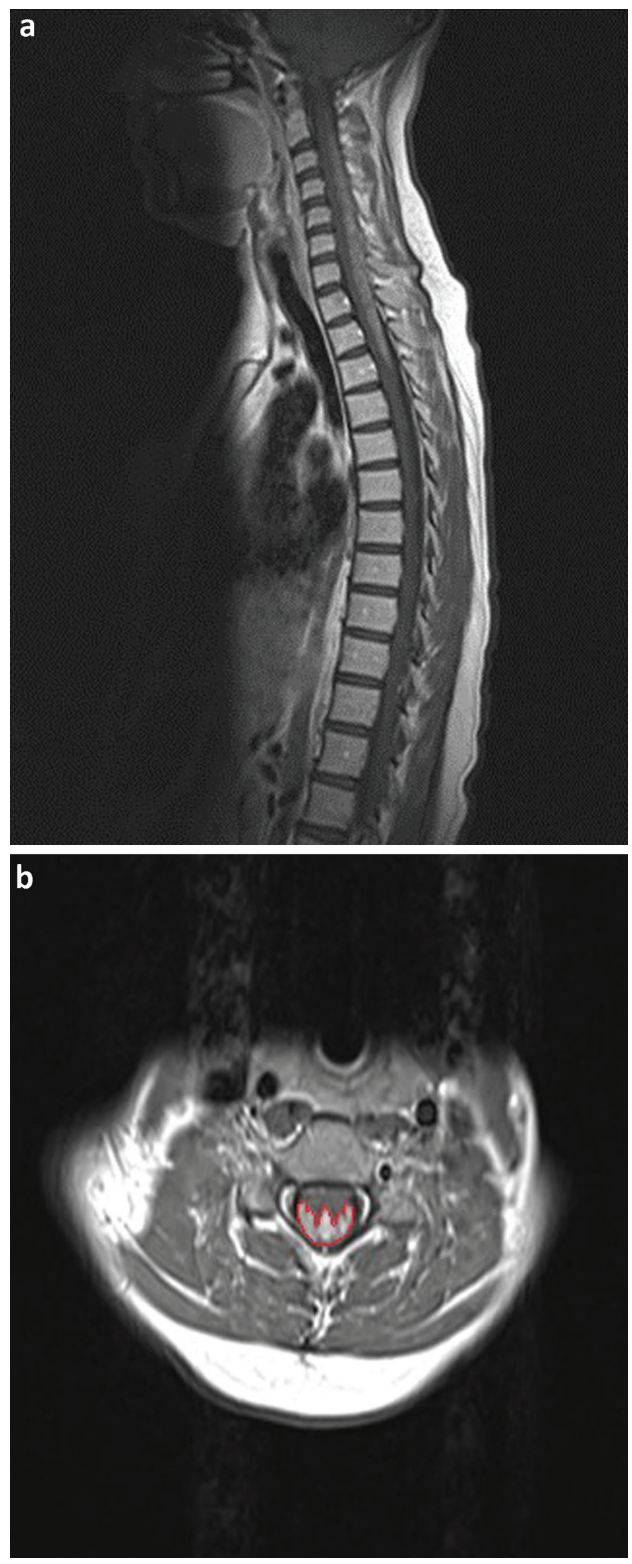

Fig 1. Magnetic resonance imaging of the spine (T1 with gadolinium). a) Sagittal view demonstrating longitudinal posterior cord lesion (dorsal subpial involvement). b) Axial view demonstrating posterior cord lesion, with the 'trident sign' highlighted. 


\section{Discussion and learning points}

When the MRI confirmed longitudinally extensive transverse myelitis (cord lesion $\geq 3$ vertebral segments), the differential diagnosis included, among others, neuromyelitis optica spectrum disorders (NMOSD), myelin oligodendrocyte glycoprotein (MOG) immunoglobulin G (IgG) associated disease, neurosarcoidosis, vascular abnormalities (eg dural arteriovenous fistula), multiple sclerosis, malignancy, and metabolic and infectious causes. ${ }^{2}$ The majority of these differentials can be confirmed or excluded with serological and/or radiological investigations.

Symptom onset (subacute), CSF findings (pleocytosis with infrequent oligoclonal bands) and steroid responsiveness may be similar between NMOSD, MOG antibody-associated disease and sarcoidosis. ${ }^{2}$ When positive, serology for aquaporin $4-\mathrm{IgG}$ and MOG-IgG is helpful, but some patients have seronegative NMOSD making the distinction from neurosarcoidosis more challenging. When considering radiological characteristics of both diagnoses, again, there are similarities: long lesion length, T1 hypointensity, cord swelling, central axial T2 hyperintensity and lesion enhancement post-gadolinium. ${ }^{2}$ The 'trident sign', evident on axial MRI, appears to be a unique radiological feature of neurosarcoidosis and a key diagnostic factor. ${ }^{1}$ The predilection for the subpial dorsal cord area, although not exclusive to this condition, is a useful diagnostic feature. This is thought to relate to the pattern of perivascular spread of leptomeningeal granulomatous inflammation along small- to medium-sized vessels and the dorsal location of the lymphatic system of the spinal meninges. ${ }^{3,4}$ The increased blood-cord permeability of the vessels in this region is also likely to be contributory. Other supportive radiological features of sarcoidosis observed in this case were subpial enhancement $\geq 2$ vertebral segments on MRI of the spine and the presence of mediastinal lymphadenopathy on the computed tomography of the chest. The history of 'adult-onset asthma' in the absence of spirometry was, in retrospect, also suspicious for an underlying systemic aetiology. CSF protein is often elevated in neurosarcoidosis but, in this case, was within normal limits. ${ }^{5}$

\section{Conclusion}

Although long-term management with immunosuppressive therapies is common to both NMOSD and sarcoidosis, careful distinction between the two conditions is clinically important due to their differing prognoses, specific treatment regimens and overall clinical course. This case highlights the importance of recognising distinct radiological features (particularly the "trident sign') in distinguishing neurosarcoidosis from other causes in presentations of subacute longitudinally extensive transverse myelitis.

\section{Conflicts of interest}

Dr Anu Jacob served on the scientific advisory board for Shire Pharmaceuticals; received travel funding and/or speaker honoraria from Biogen Idec, Shire Pharmaceuticals and Terumo BCT; consulted for Shire Pharmaceuticals; and received research support from Biogen, Alexion Pharmaceuticals, the NHS and the University of Liverpool.

\section{References}

1 Zalewski NL, Krecke KN, Weinshenker BG et al. Central canal enhancement and the trident sign in spinal cord sarcoidosis. Neurology 2016;87:743-4.

2 Flanagan EP, Kaufmann TJ, Krecke KN et al. Discriminating long myelitis of neuromyelitis optica from sarcoidosis. Ann Neurol 2016;79:437-47.

3 Jachiet V, Lhote R, Rufat P et al. Clinical, imaging, and histological presentations and outcomes of stroke related to sarcoidosis. J Neurol 2018;265:2333-41.

4 Louveau A, Herz J, Alme MN et al. CAN lymphatic drainage and neuroinflammation are regulated by meningeal lymphatic vasculature. Nat Neurosci 2018;21:1380-91.

5 Stern B], Royal W, Gelfand JM et al. Definition and consensus diagnostic criteria for neurosarcoidosis: from the Neurosarcoidosis Consortium Consensus Group. JAMA Neurol 2018;75:1546-53.

Address for correspondence: Dr Emily Gibbons, National Neuromyelitis Optica Spectrum Disorders Service. The Walton Centre NHS Foundation Trust, Lower Lane, Fazakerley, Liverpool L9 7LJ, UK.

Email: emily.gibbons@thewaltoncentre.nhs.uk 